\title{
Von Neumann Algebras Associated to Quantum-Mechanical Constants of Motion
}

\author{
A. Grossmann ${ }^{1}$ and J. Slawny ${ }^{2}$ \\ 1 Centre de Physique Théorique II, CNRS, Marseille, France \\ 2 Centre for Transport Theory and Mathematical Physics, Virginia Polytechnic Institute and \\ State University, Blacksburg, VA24061, USA
}

\begin{abstract}
We discuss, in an abstract setting, the objects which appear in the process of separating out constants of motion of "quasimomentum type" in quantum mechanics of a finite number of degrees of freedom.
\end{abstract}

\section{Introduction}

In the study of quantum-mechanical systems one almost always starts by separating out certain "trivial" constants of motion which arise because the classical Hamiltonian is invariant under a group of translations in phase space. Here are some well-known examples:

(a) $N$ isolated particles: The invariance of the energy with respect to a global shift in position is described by the equation

$$
H\left(x_{1}+a, x_{2}+a, \ldots, x_{N}+a ; p_{1}, \ldots, p_{N}\right)=H\left(x_{1}, \ldots, x_{N} ; p_{1}, \ldots, p_{N}\right)
$$

satisfied by the classical Hamiltonian. Here $a_{1} \in \mathbb{R}^{3}$, etc., for example. Similar equations hold for "cluster Hamiltonians" used in the more detailed analysis of $N$-particle systems.

(b) One particle in a periodic potential: Here the invariance condition is of the form $H(x+a, p)=H(x, p)$, where $a$ is in a discrete group, generated by three or fewer independent vectors. This leads to separation of quasimomentum ("Bloch momentum"), [3].

(c) A physically relevant example in which the Hamiltonian is invariant with respect to shifts in momentum direction is the "electronic Hamiltonian" in the theory of diatomic molecules. This is the Hamiltonian of $N+2$ interacting particles, in which the kinetic energy terms corresponding to two "heavy" particles have been suppressed.

(d) A Landau Hamiltonian, corresponding to a particle moving in a constant magnetic field, is invariant with respect to a suitable linear combination of $x$-shifts and of $p$-shifts. 
(e) This invariance is further reduced in the case of a particle subject both to a constant magnetic field and to a periodic potential. This example is discussed below, in Sect. 5 .

The aim of this paper is to discuss, at a suitable level of generality, the algebras of constants of motion that arise in such situations.

The mathematical framework that is appropriate here consists of

(i) A locally compact abelian group $E$, which plays the role of the additive group of phase space.

(ii) A multiplier $b$, and the associated bicharacter $\beta$, which brings in quantum mechanics through Weyl operator $W(g), g \in E$.

We study the von Neumann algebras generated by restrictions of the Weyl system to arbitrary closed subgroups of $E$.

\section{Notation and Terminology}

$G$ denotes an abelian, locally compact, separable group, written additively. $G^{*}$ is the dual of $G$. If $\gamma \in G^{*}$ and $g \in G$, then $\langle\gamma, g\rangle$ is the value of the character $\gamma$ on $g$.

By $b$ we shall denote a continuous multiplier on $G$, i.e. a continuous function of $G \times G$ to complex numbers of modulus one, satisfying the identities

$$
\begin{gathered}
b\left(g_{1}+g_{2}, g_{3}\right) b\left(g_{1}, g_{2}\right)=b\left(g_{1}, g_{2}+g_{3}\right) b\left(g_{2}, g_{3}\right), \\
b(0, g)=b(g, 0)=1 .
\end{gathered}
$$

A $b$-representation of $G$ is a weakly continuous map $g \mapsto W(g)$ of $G$ into unitary operators on a separable Hilbert space $H_{W}$, such that

$$
W\left(g_{1}\right) W\left(g_{2}\right)=b\left(g_{1}, g_{2}\right) W\left(g_{1}+g_{2}\right) \text {. }
$$

If $b$ is any continuous multiplier of $G$, define $\beta\left(g_{1}, g_{2}\right)=b\left(g_{1}, g_{2}\right) / b\left(g_{2}, g_{1}\right)$. Using (2.1), (2.2), one proves that $\beta$ is a character in any one of its arguments, if the other argument is kept fixed. Consequently, $\beta$ defines a map from $G$ to $G^{*}$, denoted again by $\beta,\langle\beta(g), h\rangle=\beta(g, h)$. One calls $\beta$ the associated antisymmetric bicharacter of $b$. It enters the following mostly through the identity

$$
W(g)^{-1} W(h) W(g)=\beta(h, g) W(h) \text { for any } g, h \in G .
$$

\section{Von Neumann Algebras Generated by $b$-Representations}

We first introduce a somewhat technical condition which will be satisfied in all cases of interest, as will be seen in Sect. 4.

We shall say that $a b$-representation $W$ of $G$ satisfies condition $(C)$ if there exists a weakly measurable map $\mapsto U(\gamma)$ from $G^{*}$ to unitary operators on $H_{W}$, such that

$$
U(\gamma)^{-1} W(g) U(\gamma)=\langle\gamma, g\rangle W(g) \text { for any } g \text { in } G .
$$

We shall now see that under the condition $(\mathrm{C})$ there is exactly one $b$-representation "up to multiplicity."

Define the regular $b$-representation $R$ as follows: $R$ acts on $L^{2}(G)$ - the Hilbert space of square-integrable functions with respect to a Haar measure of $G$. The 
operator $R(g)$ is defined by $(R(g) f)(h)=b(h, g) f(h+g)$. We have (cf. [4] for a more detailed proof)

3.1. Proposition. Any b-representation $W$ satisfying the condition $(\mathrm{C})$ is quasiequivalent to the regular representation $R$. That is, for every such $W$ there exist Hilbert spaces $K^{\prime}$ and $K^{\prime \prime}$ such that the b-representations $g \mapsto W(g) \otimes 1_{K^{\prime}}$ and $g \mapsto R(g) \otimes 1_{K^{\prime \prime}}$, acting in $H_{W} \otimes K^{\prime}$ and $L^{2}(G) \otimes K^{\prime \prime}$, respectively, are unitarily equivalent.

Proof. a) If $W$ is any $b$-representation of $G$ and if $g \mapsto X(g)$ is the (ordinary, abelian) regular representation of $G$ in $L^{2}(G)$, then the $b$-representation

$$
g \mapsto W(g) \otimes X(g)
$$

acting in $H_{W} \otimes L^{2}(G)$ is quasi-equivalent to $R$; this is a version of a result of Fell [5] (see [4]).

b) By a), it is sufficient to show that, under condition (C), the representation $g \mapsto W(g) \otimes X(g)$ is quasi-equivalent to $W$.

Let us identify $L^{2}(G)$ with $L^{2}\left(G^{*}\right)$ through Fourier transform, and realize the Hilbert space $H_{W} \otimes L^{2}\left(G^{*}\right)$ as the space $L^{2}\left(G^{*} ; H_{W}\right)$ of square integrable functions $f: G^{*} \rightarrow H_{W}$. The representation $g \mapsto W(g) \otimes X(g)$ takes then the form

$$
(W(g) \otimes X(g) f)(\gamma)=\langle\gamma, g\rangle W(g) f(\gamma) .
$$

Let $V$ be the unitary operator in $L^{2}\left(G^{*}, H_{W}\right)$ defined by

$$
(V f)(\gamma)=U(\gamma) f(\gamma),
$$

where $U(\gamma)$ is as in condition $(C)$, and let us calculate $V^{-1}(W \otimes 1) V$, where 1 is the identity operator in $L^{2}\left(G^{*}\right)$ :

$$
\left(V^{-1}(W(g) \otimes 1) V f\right)(\gamma)=U^{-1}(\gamma) W(g) U(\gamma) f(\gamma)=\langle\gamma, g\rangle W(g) f(\gamma),
$$

which is the same as $(W(g) \otimes X(g) f)(\gamma)$. This proves that $W$ is quasi-equivalent to $R$.

We introduce now $M(W)$, the von Neumann algebra of operators in $H_{W}$ generated by the operators $W(g), g \in G$.

3.2. Proposition. If $W$ is any $b$-representation of $G$ satisfying condition $(C)$, then $W$ is semi-finite, i.e. there exists a normal faithful semi-finite trace on $M(W)$.

Proof. Since semi-finiteness is preserved under quasi-equivalence and since we have just shown that $W$ is quasi-equivalent to the $b$-regular representation $R$, it is enough to show that $M(R)$ is semi-finite.

Semi-finiteness of $M(R)$ is obtained from the corresponding result for locally compact unimodular groups [5, Sect. 13.10]. Let $K(G)$ be the linear space of complex-valued continuous functions of compact support on $G$. One makes $K(G)$ into a Hilbert algebra by defining

$$
\begin{gathered}
(f * g)(x)=\int b(x-y, y) f(x-y) g(y) d y, \\
f^{*}(x)=\overline{b(x,-x)} f \overline{(-x)}, \quad(f \mid g)=\int f(x) \bar{g}(x) d x,
\end{gathered}
$$


where $d x$ is a Haar measure on $G$. These definitions are dictated by the requirement that for any $W$ one should have

$$
W(f) W(g)=W(f * g) \text { and } \overline{W(f)}=W\left(f^{*}\right),
$$

where

$$
W(f)=\int f(x) W(x) d x \quad(f \in K(G)) .
$$

The operation $*$ is the "twisted convolution" of [6]. As in [5, Sect. 13.10] one shows that the von Neumann algebra generated by the regular representation coincides with $V(K(G))$ of [5] and is thus semi-finite.

We ask next when the $b$-representation $W$ is not only semi-finite but finite, i.e. when $M(W)$ admits an everywhere finite normal trace. Recall that $\beta$ is a map from $G$ to $G^{*}$.

3.3. Theorem. If the set $\beta(G)$ is precompact in $G^{*}$, then every $b$-representation of $G$ satisfying condition $(\mathrm{C})$ is finite. Conversely, if there exists a finite b-representation of $G$, then $\beta(G)$ is precompact.

Proof. Assume that $\beta(G)$ is precompact, i.e. that the closure $H$ of $\beta(G)$ is a compact subgroup of $G^{*}$, and let $W$ be a $b$-representation of $G$ satisfying condition (C). Let $\varrho$ be a positive operator of trace 1 in $H_{W}$ with all eigenvalues different from zero. Define

$$
\bar{\varrho}=\int_{H} U(\gamma) \varrho U(\gamma)^{-1} d \gamma,
$$

where $U(\gamma)$ is as in condition (C), $d \gamma$ is the normalized Haar measure on $H$, and the integral is to be understood in the weak sense. This operator is also positive and of trace 1. Define $\omega(A)=\operatorname{tr}(\bar{\varrho} A)$ for any bounded operator $A$ on $H_{W}$; we shall now show that

$$
\omega\left(W\left(g_{1}\right) W\left(g_{2}\right)\right)=\omega\left(W\left(g_{2}\right) W\left(g_{1}\right)\right) \text { for all } g_{1}, g_{2} \text { in } G \text {. }
$$

This is the only nontrivial part of the proof that $\omega$ is a faithful trace. Now

$$
\begin{aligned}
\omega\left(W\left(g_{1}\right) W\left(g_{2}\right)\right) & =\operatorname{tr}\left(\varrho W\left(g_{1}\right) W\left(g_{2}\right)\right)=\int_{H} \operatorname{tr}\left(U(\gamma) \varrho U(\gamma)^{-1} W\left(g_{1}\right) W\left(g_{2}\right)\right) d \gamma \\
& =b\left(g_{1}, g_{2}\right) \int_{H} \operatorname{tr}\left(\varrho U(\gamma)^{-1} W\left(g_{1}+g_{2}\right) U(\gamma)\right) d \gamma \\
& =b\left(g_{1}, g_{2}\right) \int_{H}\left\langle\gamma, g_{1}+g_{2}\right\rangle \operatorname{tr}\left(\varrho W\left(g_{1}+g_{2}\right)\right) d \gamma \\
& =b\left(g_{1}, g_{2}\right) \operatorname{tr}\left(\varrho W\left(g_{1}+g_{2}\right)\right) \int_{H}\left\langle\gamma, g_{1}+g_{2}\right\rangle d \gamma
\end{aligned}
$$

(the interchange of the order of taking the trace and integration over $H$ is easily justified); similarly

$$
\omega\left(W\left(g_{2}\right) W\left(g_{1}\right)\right)=b\left(g_{2}, g_{1}\right) \operatorname{tr}\left(\varrho W\left(g_{1}+g_{2}\right)\right) \int_{H}\left\langle\gamma, g_{1}+g_{2}\right\rangle d \gamma .
$$

Let

$$
H^{\perp}=\left\{g \in G:\langle\gamma, g\rangle=0, \text { all } \gamma \in H^{*}\right\} ;
$$

if now $g_{1}+g_{2}$ does not belong to $H^{\perp}$ then $\int_{H} d \gamma\left\langle\gamma, g_{1}+g_{2}\right\rangle=0$ and therefore $\omega\left(W\left(g_{1}\right) W\left(g_{2}\right)\right)=\omega\left(W\left(g_{2}\right) W\left(g_{1}\right)\right)$. If on the other hand $g_{1}+g_{2} \in H^{\perp}$; then 
$\beta\left(g, g_{1}+g_{2}\right)=1$ for all $g \in G$. Setting here in particular $g=g_{1}$ and taking into account the antisymmetry of $\beta$, we see that $\beta\left(g_{1}, g_{2}\right)=1$, which is equivalent to $b\left(g_{1}, g_{2}\right)=b\left(g_{2}, g_{1}\right)$. This proves (3.4).

The converse follows from the lemma below, where the condition $(C)$ is not needed.

3.4. Lemma. If the algebra $M(W)$ has a finite (normal) trace, then $H$ is compact.

Proof. Let $\omega$ be a finite trace on $M(W)$. Consider the continuous function $\phi: g \rightarrow \omega(W(g))$ from $G$ to $C$. We note first that $\operatorname{supp}(\phi) \subset H^{\perp}$. Indeed, let $g$ not belong to $H^{\perp}$. Then there exists $g_{1} \in G$ such that $\beta\left(g, g_{1}\right) \neq 1$. We get

$$
\phi(g)=\omega(W(g))=\omega\left(W\left(g_{1}\right)^{-1} W(g) W\left(g_{1}\right)\right)=\beta\left(g, g_{1}\right) \omega(W(g))=\beta\left(g, g_{1}\right) \phi(g),
$$

which implies that $\phi(g)=0$.

Assume now that $H$ is not compact. Then $G / H^{\perp} \simeq H^{*}$ is not discrete, and therefore there exists a net of elements $g_{\alpha}$ in $G$, none of them belonging to $H^{\perp}$, converging to the identity in $G$. The continuous function $\phi$ is zero on every $g_{\alpha}$, but 1 on $e \in G$. This is a contradiction.

We now sketch a proof of the fact that $M(W)$ is approximately finitedimensional (AFD) for any $W$, i.e. that $M(W)$ is generated by an increasing sequence of its finite-dimensional von Neumann subalgebras. For a discussion of the AFD and related properties we refer to [7].

By Corollary 6.7a) of [7], any representation of an amenable discrete group generates an AFD von Neumann algebra. Let now $H$ be the Heisenberg group of $(G, b): H=G \times S^{1}$ with the group operation $(x, \lambda)(y, \mu)=(x+y, b(x, y) \lambda \mu)$.

As is well known, representations $U$ of $H$ with the property $U(0, \lambda)=\lambda 1$ are in one-to-one correspondence with $b$-representations of $G$. On the other hand, the group $H$ equipped with the discrete topology is amenable since it admits the composition series $0 \rightarrow S^{1} \rightarrow H \rightarrow G \rightarrow 0$ in which $S^{1}$ and $G$ are amenable since they are abelian, [8]. The AFD property of $M(W)$ follows.

\section{Restrictions of Perfect Systems}

Let $E$ be a locally compact separable abelian group, and $b$ a continuous multiplier of $E$. We shall say that the system $(E, b)$ is perfect if the map $\beta$ defined in Sect. 2 is a topological isomorphism of $E$ onto $E^{*}$. We note that by the open mapping theorem [9], it is enough to require here that $\beta$ yield an algebraic isomorphism from $E$ to $E^{*}$.

Let $(E, b)$ be a perfect system, let $g \mapsto W(g)$ be a $b$-representation of $E$ and let $G$ be a closed subgroup of $E$. Consider the restriction of $W$ to $G$, and the corresponding von Neumann algebra

$$
M(G)=W(G)^{\prime \prime} .
$$

4.1. Proposition. The restriction of $W$ to $G$ satisfies condition (C). Consequently all results of Sect. 3 apply to the algebra $M(G)$.

Proof. For every $g \in G$, the character $\beta(g) \in E^{*}$ restricts to a character of $G$. Conversely, by the duality theory of locally compact abelian groups, given $\gamma \in G^{*}$, 
there exists a $g \in E$ such that $\left.\beta(g)\right|_{G}=\gamma$. We have thus a continuous homomorphism from $E$ onto $G^{*}$. As is not hard to see, by [10, Chap. IX, Sect. 6, No. 8, Theorem 4], such a map has a measurable section (here we use the separability assumption on $E)$,i.e. there exists a measurable map $p$ from $G^{*}$ into $E$ such that $\left.\beta p(\gamma)\right|_{G}=\gamma$. Define now $U(\gamma)$ by

$$
U(\gamma)=W(p(\gamma))
$$

This shows then that $(C)$ is satisfied.

We now summarize the conclusions:

4.2. Let $\left(G, b_{G}\right)$ be the restriction of the perfect system $(E, b)$ to a closed subgroup $G$ of $E$; here and in the following $b_{G}$ stands for the restriction of $b$ to $G \times G$. Let $x \mapsto W(x), x \in E$, be any $b$-representation of $E$. Then the restriction of $W$ to $G$ is quasi-equivalent to the regular $b_{G}$-representation of $G$.

Remark. This does not prove that every $b$-representation of $G$ is quasi-equivalent to the regular $b$-representation; it is only a statement about the representations obtained through restrictions of perfect systems. For, by the standard argument recalled below, there exists always an irreducible $b_{G}$-representation. Thus, if the regular $b_{G}$-representation is not of type $I$, as in some situations of Sect. 5.2, there exist $b_{G}$-representations which are not quasi-equivalent to the regular one.

In the particular case $G=E$, we obtain a generalization of von Neumann's uniqueness theorem [4]:

$A$ perfect system has, up to quasi-equivalence, exactly one representation. This representation generates a factor of type $I$.

(The last statement is obtained in a standard way, [5], by an application of the Krein-Milman theorem to the set of states of the involutive algebra $L^{1}(G)$ in which the multiplication and the involution are defined as in $R(G)$ of Sect. 3.2 (see [4]): By the Krein-Milman theorem there exist extremal points in this set. These extremal points define irreducible representations of $L^{1}(G)$ and thus irreducible $b$-representations of $E$. Since all representations are quasi-equivalent each representation generates a factor of type $I$. We add that in the Mackey set-up described in Sect. 5.1, of which the usual quantum-mechanical problem is a special case, one can define the "Schrodinger representation" acting in $L^{2}(H)$, and hence obtain an irreducible representation without going through $L^{1}(G)$. We also mention that in [4] the theorem is proved without the separability assumption on $E$.)

We now ask whether the restriction $W_{G}$ generates a factor, i.e. whether $M(G)$ is a factor.

As earlier, we denote by $G^{\perp} C E$ the set of all $g \in E$ such that $\beta(g, h)=1$ for every $h \in G$. Furthermore, denote by $\beta_{G}$ the restriction of $\beta$ to $G \times G$. Let

$$
\operatorname{Ker}\left(\beta_{G}\right)=\{g \in G: \beta(g, h)=1, \text { for all } h \in G\}
$$

we say that $\beta_{G}$ is nondegenerate if $\operatorname{Ker}\left(\beta_{G}\right)=\{0\}$. One can prove by elementary considerations the following. 
4.3. Lemma. The following conditions are equivalent:

i) $G \cap G^{\perp}=\{0\}$,

ii) $G+G^{\perp}$ is dense in $E$,

iii) $\beta_{G}$ is nondegenerate,

iv) $\beta_{G^{\perp}}$ is nondegenerate,

v) $\beta_{G}(G)$ is dense in $G^{*}$,

vi) $\beta_{G^{\perp}}\left(G^{\perp}\right)$ is dense in $\left(G^{\perp}\right)^{*}$.

Note that the definition of $G^{\perp}$ involves $\beta$.

4.4. Theorem. The von Neumann algebra $M(G)$ is a factor if and only if $G$ satisfies any one of the conditions of the preceding lemma.

Proof. Since $W\left(\operatorname{Ker}\left(\beta_{G}\right)\right)$ is contained in the center of $M(G)$, if $\beta_{G}$ is degenerate, then obviously $M(G)$ is not a factor. On the other hand, elements of the center of $M(G)$ commute with $W\left(G+G^{\perp}\right)$ which in the nondegenerate case generates the same von Neumann algebra as $W(E)$ (preceding lemma).

4.5. Theorem. Suppose $M(G)$ is a factor, i.e. $\operatorname{Ker}\left(\beta_{G}\right)=\{0\}$. Then $M(G)$ is of type $I$ if and only if $\beta_{G}(G)=G^{*}$, i.e. if $\left(G, \beta_{G}\right)$ is perfect.

Proof. We have already seen that a perfect system yields a factor of type $I$. It remains to show that if $M(G)$ is a factor of type $I$ then $\left(G, \beta_{G}\right)$ is perfect.

Let $M_{0}$ be the linear span of $\{W(x): x \in G\}$; because of the commutation relations, $M_{0}$ is a dense *-subalgebra of $M=M(G)$ ( $M$ equipped with, say, the weak topology). The proof will be in three steps; the last one contains the heart of the matter.

Assume $M$ to be a type $I$ factor.

a) For any $\xi \in G^{*}$ there is a unitary $U(\xi) \in M$, unique up to a numerical factor, such that

$$
U(\xi)^{-1} W(x) U(\xi)=\langle\xi, x\rangle W(x), \text { for all } x \in G .
$$

Let $\xi \in G^{*}$ and let $y_{\xi} \in E$ be such that

$$
\beta\left(x, y_{\xi}\right)=\langle\xi, x\rangle, \text { all } x \in G ;
$$

such a $y_{\xi}$ exists since $\beta$ is perfect and $E^{*} \rightarrow G^{*}$ is surjective. Then

$$
W\left(y_{\xi}\right)^{-1} W(x) W\left(y_{\xi}\right)=\langle\xi, x\rangle W(x), \text { all } x \in G,
$$

and by density of $M_{0}$ in $M, W\left(y_{\xi}\right)^{-1} A W\left(y_{\xi}\right) \in M$ for all $A \in M$. Thus $A \mapsto W\left(y_{\xi}\right)^{-1} A W\left(y_{\xi}\right)$ is an automorphism of $M$, and since $M$ is a factor of type $I$ the existence and required properties of $U(\xi)$ follow.

b) By going to a quasi-equivalent representation of $M$ we can assume that $M$ is the algebra $L(K)$ of all bounded operators on a separable Hilbert space $K$; we retain the notation $W(x)$ and $U(\xi)$ for the corresponding operators in the new representation. Let $L^{2}(K)$ be the (separable) Hilbert space of the Hilbert-Schmidt operators on $K$ with the scalar product $(A \mid B)=\operatorname{tr}\left(A^{*} B\right), A, B \in L^{2}(K)$. For $\xi \in G^{*}$, let $V_{\xi}$ be the unitary operator on $L^{2}(K)$ defined by $V_{\xi}(A)=U(\xi)^{-1} A U(\xi)$. Clearly $\xi \mapsto V_{\xi}$ is a unitary representation of $G^{*}$ on $L^{2}(K)$ which we will now show to be (weakly) continuous. 
By the well-known result of Segal and von Neumann $[5,13.11 .8]$ it is enough to show that for any $A, B \in L^{2}(K)$ the function $\xi \mapsto\left(A \mid V_{\xi} B\right)$ is measurable with respect to the Haar measure of $G^{*}$. Now, for $\Phi, \Psi \in K$ and $A \in M_{0}$, the function $\xi \mapsto\left(\Phi \mid U(\xi)^{-1} A U(\xi) \Psi\right)$ is obviously continuous. Since any element of $M$ is a weak limit of a sequence of elements of $M_{0}$ (by separability of $K$ and the Kaplansky density theorem), $\xi \mapsto\left(\Phi \mid U(\xi)^{-1} A U(\xi) \Psi\right)$ is a measurable function for any $A \in L(K)$. If now $B$ is the rank 1 operator $\Theta \mapsto(\Psi \mid \Theta) \Phi$ and $A \in L^{2}(K)$, then

$$
\left(B \mid V_{\xi} A\right)=\operatorname{tr}\left(B^{*} U(\xi)^{-1} A U(\xi)\right)=\left(\Phi \mid U(\xi)^{-1} A U(\xi) \Psi\right),
$$

and thus the function $\xi \mapsto\left(B \mid V_{\xi} A\right)$ is measurable. Since linear combinations of rank 1 operators are dense in the (separable) Hilbert space $L^{2}(K)$, by taking limits one obtains measurability of $\xi \mapsto\left(B \mid V_{\xi} A\right)$ for any $B, A \in L^{2}(K)$. Thus weak continuity of $\xi \mapsto V_{\xi}$ has been demonstrated.

c) If $\xi=\beta_{G}(x), x \in G$, then by uniqueness of $U(\xi)$,

$$
V_{\xi} A=W(x)^{-1} A W(x) .
$$

Suppose now that $\xi \in G^{*} \backslash \operatorname{Im}\left(\beta_{G}\right)$. Since $\operatorname{Im}\left(\beta_{G}\right)$ is dense in $G^{*}$, there exists a sequence $\left(x_{n}\right) \subset G$ such that $\xi_{n}=\beta_{G}\left(x_{n}\right) \rightarrow \xi$ as $n \rightarrow \infty$. We claim that $x_{n} \rightarrow \infty$ as $n \rightarrow \infty$, i.e. that for any compact $C \subset G$ there is a natural number $n_{C}$ such that $x_{n}$ is not in $C$ for $n>n_{C}$. For otherwise there would exist a subsequence, say $\left(x_{n_{m}}\right)$, of $\left(x_{n}\right)$ convergent in $G$ to, say, $x$. By the strong continuity of $x \mapsto W(x)$ and $\xi \mapsto V_{\xi}$ it would follow that $V_{\xi} A=W(x)^{-1} A W(x)$. Hence since $\xi \rightarrow V_{\xi}$ is one-to-one, $\xi \in \operatorname{Im}\left(\beta_{G}\right)$, in contradiction to the assumption. Thus $x_{n} \rightarrow \infty$.

However, $W\left(x_{n}\right)$ tends weakly to 0 as $x_{n} \rightarrow \infty$. This is obvious in the regular $b_{G}$-representation of $G$ since in this representation $W(x)$ shifts by $x$ and multiplies by a function of modulus 1 , and in our representation it follows from its quasiequivalence to the regular one and from the invariance of the weak topology on bounded sets under isomorphisms of von Neumann algebras. Thus if $A: \Theta \mapsto(\Psi \mid \Theta) \Phi$ and $B: \Theta \mapsto\left(\Psi^{\prime} \mid \Theta\right) \Phi^{\prime}$ are rank 1 operators then

$$
\left(B \mid V_{\xi_{n}} A\right)=\operatorname{tr}\left(B^{*} W\left(x_{n}\right)^{-1} A W\left(x_{n}\right)\right)=\left(W\left(x_{n}\right) \Phi^{\prime} \mid \Phi\right)\left(\Psi \mid W\left(x_{n}\right) \Psi^{\prime}\right) \rightarrow 0
$$

as $n \rightarrow \infty$. By density of linear combinations of rank 1 operators in $L^{2}(K)$ we obtain that $V_{\xi_{n}} \rightarrow 0$ (weakly) as $n \rightarrow \infty$, which contradicts the continuity of $\xi \mapsto V_{\xi}$, the unitaritity of $V_{\xi}$ and the fact that $\xi_{n} \rightarrow \xi$.

Summarizing, we have

4.6. Theorem. The von Neumann algebra $M(G)$ obtained by restricting a perfect system $(E, b)$ to a closed subgroup $G$ is a factor if and only if $G$ satisfies any one of the conditions of Lemma 4.3. The factor is of type I if and only if $\beta_{G}(G)=G^{*}$ or, equivalently, if $G+G^{\perp}=E$. It is of type II if $\beta_{G}(G) \neq G$ and $\beta_{G}(G)$ has compact closure in $G$. It is of type $I I_{\infty}$ in all other cases.

Remark. Not only are the algebras semi-finite but each of them carries a natural (normal) trace: by the proof of Proposition 3.1, the map $W(x) \mapsto R(x)$ extends to an isomorphism from $M(G)$ to $M(R)$. However, $M(R)$ carries the natural trace of the standard von Neumann algebra of the Hilbert algebra $K(G)$. In particular, it is not hard to deduce that if $\phi=\phi_{1} * \phi_{2}, \phi_{1} \in K(G), \phi_{2} \in K(G)$, then $W(\phi)=\int W(x) \phi(x) d x$ is of finite trace and $\omega(W(\phi))=\phi(0)$. This generalizes well known facts about $M(E)$. 


\section{Examples}

\subsection{Perfect Systems}

If $(E, b)$ is a perfect system, then $E$ must be isomorphic to its dual. This is certainly the case if $G$ is of the form $G=H+H^{*}$, where $H$ is locally compact abelian and $H^{*}$ is the dual of $H$. A natural nontrivial multiplier is then

$$
b\left(h, \xi ; h^{\prime}, \xi^{\prime}\right)=\left\langle\xi, h^{\prime}\right\rangle \quad\left(h, h^{\prime} \in H ; \xi, \xi^{\prime} \in H^{*}\right),
$$

with the associated antisymmetric bicharacter

$$
\beta\left(h, \xi ; h^{\prime}, \xi\right)=\left\langle\xi, h^{\prime}\right\rangle\left\langle\left\langle\xi^{\prime}, h\right\rangle .\right.
$$

This case has been analyzed by Mackey [2]; we do not know whether each perfect system is equivalent to one of the above form. The irreducible representation of a perfect system is unique up to unitary equivalence. Its Hilbert space can be considered as the space of pure states for the corresponding quantummechanical system.

\subsection{Non-Perfect Systems}

Particle in periodic potential and magnetic field. We consider a quantummechanical particle in three dimensions, subject to a periodic local potential $V$ and a constant magnetic field $B$ of arbitrary strength and direction. The phase space is $E=\mathbb{R}^{3}+\mathbb{R}^{3}$ and $\beta$ is expi $\sigma$, where the symplectic form $\sigma$ is given by $\sigma\left(x, p \mid x^{\prime}, p^{\prime}\right)$ $=x p^{\prime}-p x^{\prime}$. Hence we have a perfect system of the Mackey type.

The Hamiltonian of our system is in suitable units

$$
H=(p-x \wedge B)^{2}+V(x),
$$

with $V(x+a)=V(x)$ for all $a \in L$, where $L$ is a lattice in $x$-space. Denote by $\Gamma$ the corresponding reciprocal lattice $\Gamma=\{K: K \cdot a$ is integer for all $a \in L\}$.

The classical function $H(x, p)$, given by $(5.3)$ is invariant with respect to a discrete group of phase-space translations: indeed, for every $a \in L$, we have

$$
H(x+a, p+a \wedge B)=H(x, p) .
$$

The Weyl operators corresponding to these translations $W(a, a \wedge B), a \in L\left(\simeq Z^{3}\right)$, all commute with $H$ but do not, in general, commute with each other.

For $V$ satisfying suitable regularity assumptions, by general arguments which are omitted, $H$ is associated to the von Neumann algebra $M(G)$ where $G$ is the subgroup of phase space defined as

$$
G=\left\{(x, B \wedge x+k), x \in R^{3}, k \in \Gamma\right\} \quad\left(G \simeq R^{3} \times Z^{3}\right) .
$$

The restriction of the symplectic form $\sigma$ to $G$ is

$$
\sigma_{G \times G}\left(x, k \mid x^{\prime}, k^{\prime}\right)=-2 B \cdot\left(x \wedge x^{\prime}\right)+x \cdot k^{\prime}-k \cdot x^{\prime} .
$$

Applying the results of Sect. 4, we obtain the following generalization of the "irrational flux" conditions:

Theorem. Consider, in momentum space, the lattice $B \wedge$ Lobtained from the lattice $L$ in $x$-space by outer multiplication with $B$. If $B \wedge L$ has trivial intersection with the 
reciprocal lattice $\Gamma$ (i.e. if $(B \wedge L) \cap \Gamma=\{0\})$, then $M(G)$ is of type $I I_{\infty}$. If $(B \wedge L) \cap \Gamma \neq\{0\}$, then $M(G)$ is of type $I I$.

Acknowledgements. One of the authors (J.S.) would like to thank Centre de Physique Théorique CNRS (Marseille) for warm hospitality. His work on this paper has been supported in part by the NSF grant MCS 8301709.

\section{References}

1. von Neumann, J.: Math. Ann. 104, 570 (1931)

2. Mackey, G.W.: In: Functional analysis and related fields. Browder, F. (ed.). Berlin, Heidelberg, New York: Springer 1968

3. Grossmann, A.: In: Statistical mechanics and field theory. Sen, R.N., Weil, C. (eds.). New York: Halsted Press 1972

4. Slawny, J.: On the regular representation, von Neumann uniqueness theorem, and the 22-algebra of canonical commutation and anticommutation relations, Preprint TAUP227 71, Tel Aviv University; and Simon, B.: pp. 67-76. In: Mathematics of contemporary physics. Streater, R. (ed.). New York: Academic Press 1972

5. Dixmier, J.: Les $C^{*}$-algèbres et leurs représentations. Paris: Gauthier-Villars 1964

6. Kastler, D.: The $C^{*}$-algebras of a free boson field. I. Discussion of the basic facts. Commun. Math. Phys. 1, 14 (1968)

7. Connes, A.: Ann. Math. 109, 73 (1976)

8. Ruelle, D.: Statistical mechanics. New York: Benjamin 1969

9. Pontryagin, L.S.: Topological groups. New York: Gordon and Breach 1966

10. Bourbaki, N.: General topology. Paris: Hermann 1966

Communicated by $\mathrm{H}$. Araki

Received November 25, 1985; in revised form August 6, 1986 